\title{
Synaptic Islands Defined by the Territory of a Single Astrocyte
}

\author{
Michael M. Halassa, ${ }^{\star}$ Tommaso Fellin ${ }^{\star}$ Hajime Takano, Jing-Hui Dong, and Philip G. Haydon \\ Silvio Conte Center for Integration at the Tripartite Synapse, Department of Neuroscience, University of Pennsylvania School of Medicine, Philadelphia, \\ Pennsylvania 19104
}

In the mammalian brain, astrocytes modulate neuronal function, in part, by synchronizing neuronal firing and coordinating synaptic networks. Little, however, is known about how this is accomplished from a structural standpoint. To investigate the structural basis of astrocyte-mediated neuronal synchrony and synaptic coordination, the three-dimensional relationships between cortical astrocytes and neurons was investigated. Using a transgenic and viral approach to label astrocytes with enhanced green fluorescent protein, we performed a three-dimensional reconstruction of astrocytes from tissue sections or live animals in vivo. We found that cortical astrocytes occupy nonoverlapping territories similar to those described in the hippocampus. Using immunofluorescence labeling of neuronal somata, a single astrocyte enwraps on average four neuronal somata with an upper limit of eight. Single-neuron dye-fills allowed us to estimate that one astrocyte contacts $300-600$ neuronal dendrites. Together with the recent findings showing that glial $\mathrm{Ca}^{2+}$ signaling is restricted to individual astrocytes in vivo, and that $\mathrm{Ca}^{2+}$ signaling leads to gliotransmission, we propose the concept of functional islands of synapses in which groups of synapses confined within the boundaries of an individual astrocyte are modulated by the gliotransmitter environment controlled by that astrocyte. Our description offers a new structurally based conceptual framework to evaluate functional data involving interactions between neurons and astrocytes in the mammalian brain.

Key words: gliotransmission; astrocyte; contact; cortex; spine; dendrite

\section{Introduction}

Astrocytes release neuroactive molecules, which influence neuronal excitability and synaptic transmission: glutamate (Parpura et al., 1994; Bezzi et al., 1998) controls synaptic strength (Fiacco and McCarthy, 2004; Kang et al., 1998; Liu et al., 2004), whereas D-serine (Schell et al., 1995) regulates metaplasticity (Panatier et al., 2006). The astrocytic release of ATP (Arcuino et al., 2002) causes a presynaptic inhibition of excitatory synaptic transmission, an action that is mediated by adenosine, which accumulates after the hydrolysis of released ATP (Zhang et al., 2003; Pascual et al., 2005; Serrano et al., 2006). Stimulation of a restricted set of synaptic terminals causes the activity-dependent release of additional ATP from the astrocyte which, after hydrolysis to adenosine, causes a heterosynaptic depression of neighboring synapses. Although gliotransmitters can have these actions, there is little information on the structural interrelationship between neurons and astrocytes that permits an astrocyte to coordinate groups of synapses or neurons.

Electron microscopy has shown that astrocytes contact neuronal somata, dendrites, spines, and presynaptic terminals (Ven-

Received March 29, 2007; revised May 7, 2007; accepted May 9, 2007

This work was supported by National Institutes of Health Grants NS-054770, MH-071705, NS-037585, NS043142, and NS-047321. We thank Dr. Munoz for comments on this manuscript and Elizabeth Stambrook and Huan-Ying Zhu for help with the transgenic colony.

${ }^{*}$ M.M.H. and T.F. contributed equally to this work.

Correspondence should be addressed to Philip G. Haydon, Department of Neuroscience, University of Pennsylvania School of Medicine, Philadelphia, PA 19104.E-mail: pghaydon@mail.med.upenn.edu.

DOI:10.1523/JNEUROSCI.1419-07.2007

Copyright $\odot 2007$ Society for Neuroscience $\quad$ 0270-6474/07/276473-05\$15.00/0 tura and Harris, 1999). Dye filling of adjacent hippocampal astrocytes has shown that astrocytes are organized in a nonoverlapping tile-like manner and that one hippocampal astrocyte can contact in excess of 100,000 synapses (Bushong et al., 2002). Glutamate released from astrocytes can synchronize closely spaced neurons through the generation of slow inward currents (SICs) (Angulo et al., 2004; Fellin et al., 2004; Kozlov et al., 2006). Neuronal somata separated by $<100 \mu \mathrm{m}$ display synchronous SICs. Confocal imaging of $\mathrm{Ca}^{2+}$ indicators reveals that an average of two to three, but up to 12 , hippocampal pyramidal neurons per optical section are synchronously activated by glutamate released from astrocytes (Fellin et al., 2004).

Although these studies are illuminating the structural basis for neuron-glial interactions, we have almost no idea about the numbers of neurons that one astrocyte can influence. Using capricious expression of enhanced green fluorescent protein (EGFP) together with double-labeling for anti-NeuN and patch pipette-mediated single-neuron dye filling, we show that cortical astrocytes occupy nonoverlapping domains, and that a single cortical astrocyte enwraps, on average, four neuronal cell bodies and hundreds of dendrites. Thus, an astrocyte has the potential to coordinate small clusters of neurons if signals are propagated directly between an astrocyte and neuronal cell bodies, whereas when signals are transmitted from astrocytes to dendrites/spines/ synapses, one astrocyte has the potential to regulate the function of hundreds of neurons with spatially dispersed somata. Based on this structural relationship, we propose an additional layer of nervous system organization in which clusters of synapses are 
coordinated at nodes that correspond to the domain of individual astrocytes.

\section{Materials and Methods}

Transgenic line generation. We used previously developed lines of transgenic animals (Pascual et al., 2005). The GFAP.tTA line contains the human GFAP promoter that drives the expression of the tet-off tetracycline transactivator. tetO.dnSNARE contains three transgenes: tetO.dnSNARE (dnSNARE corresponds to amino acids $1-96$ of synaptobrevin II), tetO.lacZ and tetO.GFP both being reporters. To prevent potential developmental influences of transgenes, animals were maintained on a diet including doxycycline (dox) until weaning. In these studies, dox was removed from the diet for at least 2 weeks before investigating the structure of the astrocyte.

Tissue processing and confocal imaging. Six- to 12-week-old transgenic mice were anesthetized with halothane and perfused transcardially with $4 \%$ paraformaldehyde. Brains were postfixed (30 min) and placed in 30\% sucrose overnight. Brains were frozen and $40 \mu \mathrm{m}$ sections were cut on a freezing microtome. For immunostaining, free-floating sections were permeabilized with $0.1 \%$ Triton $\mathrm{X}-100,5 \%$ goat serum in PBS. Rabbit GFAP antibody (Sigma, St. Louis, MO), 1:1000, or mouse NeuN antibody (Millipore, Temecula, CA), 1:1000, were used for 48 and $24 \mathrm{~h}$, respectively. Secondary antibodies conjugated to Alexa 546 were subsequently used. Sections were mounted and visualized on a confocal microscope (Olympus, Center Valley, PA).

Virus generation and injection. To control for potential effects of dnSNARE expression in transgenic animals, we also used adenoassociated virus, which was obtained from the University of Pennsylvania Gene Therapy Virus Core. The CMV.EGFP3 transgene cassette was used and virus was generated using a $2 / 5$ serotype. This serotype has preferential tropism for astrocytes (J. Dong, unpublished observations). Virus $\left(1.55 \times 10^{10}\right.$ viral particles/ $\left.\mu \mathrm{l}\right)$ was determined to be free of endotoxin. Two microliters were stereotaxically injected at the following coordinates into isoflurane-anesthetized eight-week-old wild-type FVB/N mice: anteroposterior, $-1.0 \mathrm{~mm}$; mediolateral, $\pm 1.0 \mathrm{~mm}$; dorsoventral, -1.0 $\mathrm{mm}$. EGFP expression was examined $7 \mathrm{~d}$ later.

In vivo two-photon microscopy. Six- to 8-week-old animals were anesthetized with urethane ( $2 \mathrm{~g} / \mathrm{kg}$ of body weight) and placed in a stereotaxic apparatus. The level of anesthesia was monitored by checking reflexes to tail and paw pinching. Body temperature was monitored and maintained at $37^{\circ} \mathrm{C}$ with a rectal probe (Harvard Apparatus, Holliston, MA). A craniotomy $(1.8 \mathrm{~mm})$ was drilled in the skull and the dura carefully removed. The cortical surface was kept moist with normal HEPES-buffered artificial CSF (ACSF). A metal plaque was sealed on the skull and a glass coverslip was glued on top of the craniotomy. The space between the cortical surface and the glass coverslip was filled with $2 \%$ agarose in ACSF. An Ultima two-photon microscope (Prairie Technologies, Middleton, WI) equipped with a $40 \times$ water-immersion objective was used for in vivo visualization of EGFP-expressing astrocytes. Excitation for EGFP was provided at $820-920 \mathrm{~nm}$ from a Coherent (Santa Clara, CA) Chameleon laser and emission was detected by external photomultiplier tubes.

Brain-slice preparation and biocytin filling and staining. Mice were anesthetized with halothane, the brain was rapidly removed and slices $(300 \mu \mathrm{m})$ were cut transversally while maintained in cold cutting solution containing (in mM) $120 \mathrm{NaCl}, 3.2 \mathrm{KCl}, 1 \mathrm{KH}_{2} \mathrm{PO}_{4}, 26 \mathrm{NaHCO}_{3}, 2$ $\mathrm{MgCl}_{2}, 1 \mathrm{CaCl}_{2}, 10$ glucose, $2 \mathrm{Na}$-pyruvate, and 0.6 ascorbic acid at $\mathrm{pH}$ 7.4 with $\mathrm{O}_{2} 95 \%, \mathrm{CO}_{2} 5 \%$. After $1 \mathrm{~h}$ at $37^{\circ} \mathrm{C}$, slices were placed in the recording chamber and perfused with ACSF containing (in mM) 120
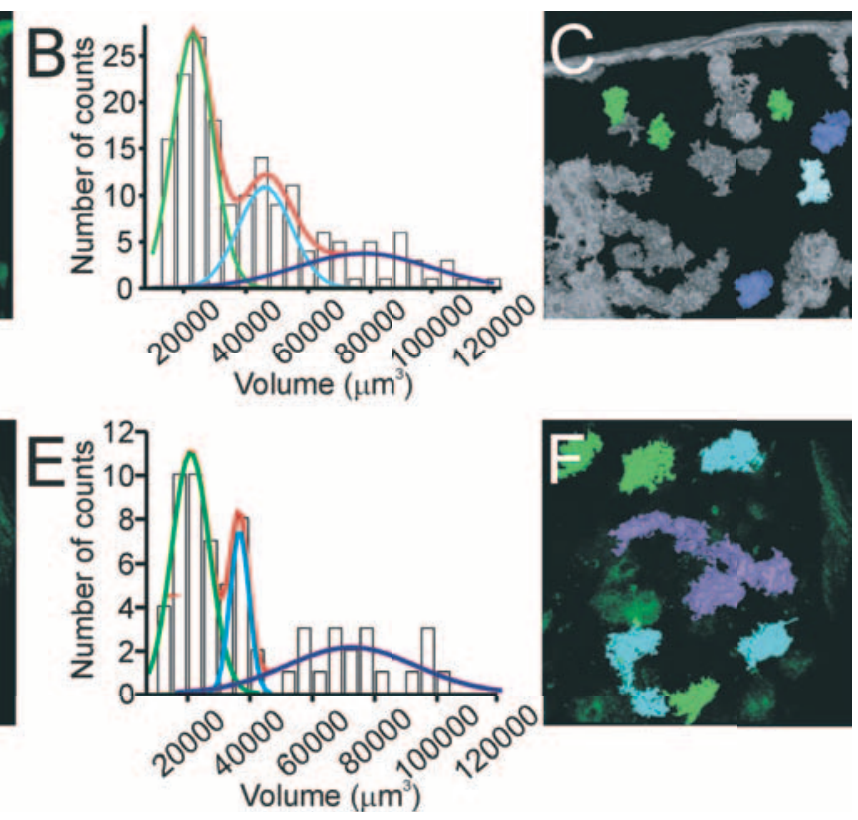

Volume $\left(\mu \mathrm{m}^{3}\right)$

Figure 1. Cortical astrocytes occupy nonoverlapping domains. A, Top-view reconstruction of EGFP-expressing cortical astroctes from dnSNARE coronal brain sections. $\boldsymbol{B}$, Histogram representing the volume distribution of EGFP labeling of astrocytes. Raw astrocyte volume distribution shows three distinct peaks at 21,033, 36,713 and 72,664 ( $n=3$ dnSNARE animals). $\boldsymbol{F}$, Using the , structures corresponding to single, two, and three astrocytes are shown.

$\mathrm{NaCl}, 3.2 \mathrm{KCl}, 1 \mathrm{KH}_{2} \mathrm{PO}_{4}, 26 \mathrm{NaHCO}_{3}, 1 \mathrm{MgCl}_{2}, 2 \mathrm{CaCl}_{2}$, and 10 glucose at $\mathrm{pH} 7.4$ with $\mathrm{O}_{2} 95 \%, \mathrm{CO}_{2} 5 \%$. Biocytin filling of cortical neurons was achieved by patch-clamping the cell (pipette resistance, 3-4 M $\Omega$ ) in whole-cell configuration for at least $30 \mathrm{~min}$. Intrapipette solutions were 140 K-gluconate, $1 \mathrm{MgCl}_{2}, 8 \mathrm{NaCl}, 2 \mathrm{Na}$-ATP, $0.5 \mathrm{Na}$-GTP, 10 HEPES, and 10 phosphocreatine, pH 7.2 with $\mathrm{KOH}$, or $140 \mathrm{CsCl}, 8 \mathrm{NaCl}, 2$ Na-ATP, 0.5 Na-GTP, 10 HEPES, 10 phosphocreatine, and 2.5 QX-314 [N-(2,6-dimethylphenylcarbamoylmethyl) triethyl ammonium], $\mathrm{pH} 7.2$ with $\mathrm{CsOH}$. Biocytin $(2 \mathrm{mg} / \mathrm{ml})$ was added to the solutions before recording. Slices were fixed overnight in paraformaldehyde (4\% in PBS) at $4^{\circ} \mathrm{C}$, then rinsed several times with PBS and incubated with PBS $/ 0.4 \%$ Triton X-100 and 1:250 streptavidin Alexa 546 for $4 \mathrm{~h}$ at room temperature.

Data analysis. To measure the volume of a single astrocyte, $1 \mu \mathrm{m}$ step confocal $z$-series of either dnSNARE brain sections or adeno-associated virus 2/5 (AAV)-injected EGFP-labeled brain sections were analyzed using Volocity (Improvision, Lexington, MA) software. Images were binarized and the volume of EGFP-expressing astrocytes was calculated. Cells contacting an edge of the field of view were excluded from analysis, as were volumes of $<10,000 \mu \mathrm{m}^{3}$ because they represented cellular structures that were cut during sectioning. For AAV analysis, only structures that were confirmed to be single astrocytes (with GFAP labeling) were included.

To measure the number of neurons enwrapped by a single astrocyte, 1 $\mu \mathrm{m} z$-series of dnSNARE sections stained with NeuN were used. We first identified a single astrocyte based on the volume analysis described previously. We then identified each NeuN-labeled neuron with at least 50\% of its surface covered by an astrocyte and determined the number of these neurons that contacted a single astrocyte.

In some studies, we performed a nearest-neighbor analysis on neuronal somata to ask whether neurons clustered within the territory of a single astrocyte. The center of each neuron within the proximity of a single astrocyte was identified and its $x, y$, and $z$ coordinates were recorded. An algorithm written in LabView (National Instruments, Austin, TX) was used to determine the shortest distance for each neuron enwrapped by an astrocyte to its neuronal neighbors. Nearest neighbors 

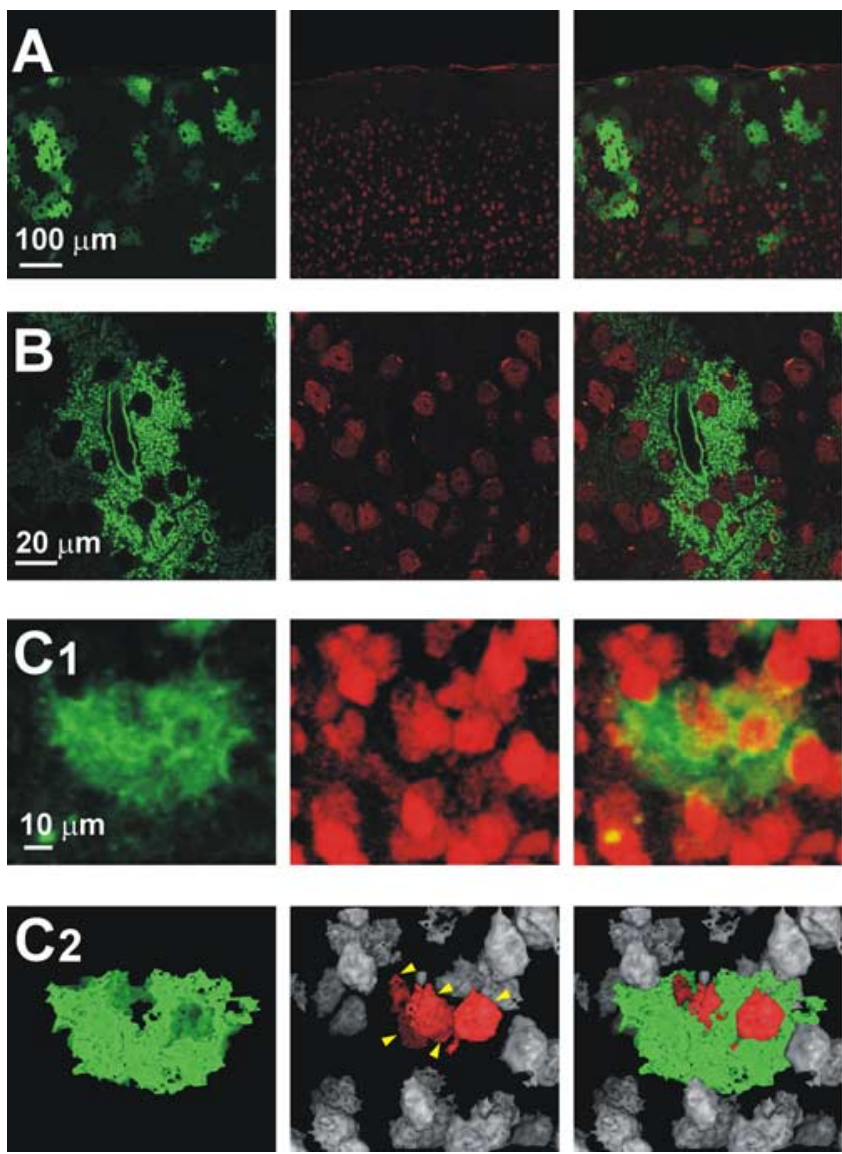

Figure 2. One astrocyte enwraps several neuronal somata. $\boldsymbol{A}$ and $\boldsymbol{B}$ show individual optical sections to reveal the structure of astrocytes expressing EGFP within the dnSNARE mouse (green; left), the location of neurons immunolabeled with anti-NeuN (red; middle), and an overlay (right). Note that the astrocytes contain several holes, which correspond with the locations of neuronal somata. C1, Top-view reconstruction of a single astrocyte (green) and neurons (red). C2, The same cells shown in C1 are depicted in a top-view reconstruction with a threshold mask. Neurons within the astrocytic territory are red (yellow arrows). D, Distribution of the number of neuronal somata that contact one astrocyte. At least $50 \%$ of the surface area of a neuron had to make contact with the astrocyte to be considered as having sufficient interaction for inclusion in this analysis. $\boldsymbol{E}, \boldsymbol{F}, \mathrm{A}$ single confocal plane of an AAV-transduced astrocyte (green) contacting neuronal somata (red). $\boldsymbol{G}$, Three-dimensional reconstruction of the astrocyte in $\boldsymbol{E}$ and $\boldsymbol{F}$.

were identified and we then determined whether these neighbors were located within the same astrocytic territory (supplemental Fig. 3, available at www.jneurosci.org as supplemental material).

To measure the length of neuronal dendrite covered by an astrocyte, confocal $z$-series containing the biocytin-filled neuron and EGFPpositive astrocytes were studied using Volocity software. Single astrocytes were identified as mentioned previously. Of the nine layer $2 / 3$ neurons that were successfully loaded with and stained for biocytin, only four were positioned in close proximity to single EGFP+ astrocytes. In these four neurons, 24 dendrites were observed to span through single EGFP+ astrocytes.

To calculate spine density, horizontally projecting dendrites from nine layer $2 / 3$ neurons were visualized at high magnification $(z$-stack acquisition, $60 \times 1.4$ numerical aperture objective and 2.1 digital zoom). One hundred and five regions of dendrites of variable length (between 9 and $72 \mu \mathrm{m}$ ) were considered for analysis. The distance of the analyzed dendrite from the parent cell body was always $<200 \mu \mathrm{m}$. A spine was defined as a structure with a clear fluorescence head connected to the dendrite by the thin neck. Filopodia-like spines were not considered in the analysis. Spines positioned above and underneath the dendritic shaft were not clearly identified and, thus, excluded from the analysis.

\section{Results}

Cortical astrocytes occupy nonoverlapping territories

As a first step in studying the structure of individual astrocytes, we determined the degree to which the processes of an astrocyte
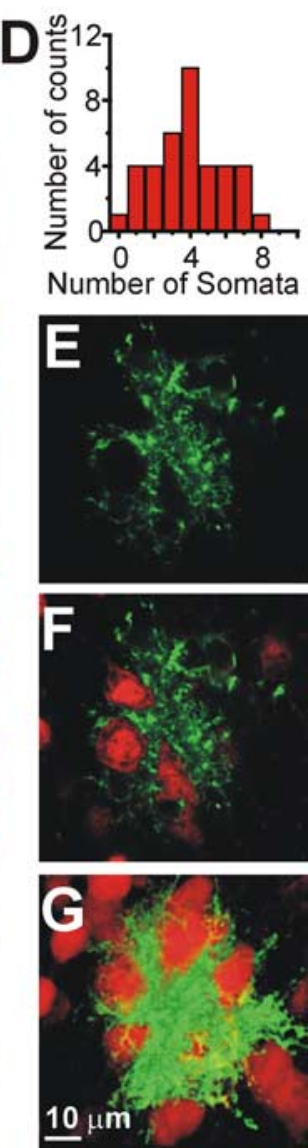

overlap one another. We cut sections from a bigenic transgenic animal (GFAP.tTA $\times$ tetO.dnSNARE) (Pascual et al. 2005) in which EGFP is expressed selectively within astrocytes (see Materials and Methods). Because EGFP is not expressed in all astrocytes, it was possible to determine the volume occupied by EGFP when few cells express the reporter transgene (Fig. $1 A$ ). We selected volumes for analysis in which there were isolated patches of fluorescence with the expectation that they would correspond to EGFP expression in few astrocytes. The volume distribution showed three distinct peaks at 22,906, 46,188, and $78,684 \mu \mathrm{m}^{3}$ (Fig. $1 \mathrm{~B}$ ), consistent with a quantal nature of volumes containing EGFP. To test whether the volume measurements were affected by sectioning and tissue processing, we performed in vivo two-photon imaging of EGFP expression in the cortex of dnSNARE mice (Fig. 1D). In agreement with data obtained from tissue sections, we found that EGFP-positive structures had distinct peaks on a volume distribution at 21,033, 36,713 and 72,664. $\mu \mathrm{m}^{3}$ (Fig. $1 E, F$ ).

Because of the apparent quantal nature of this distribution, which suggests that each mode corresponds to the expression of EGFP in one, two, and three adjacent but nonoverlapping astrocytes, we asked whether volumes of EGFP fluorescence that were contained within the first peak contained only one astrocyte by double labeling with anti-GFAP. In all EGFP volumes examined within the first mode of the distribution, we found the soma of only one astrocyte (supplemental Fig. 1, available at www. jneurosci.org as supplemental material).

The dnSNARE animals express multiple transgenes within astrocytes, raising the potential that nonreporter genes modify the normal structure of the astrocyte. We therefore used an independent assessment of astrocyte volume which was based on viralmediated expression of EGFP alone. We stereotaxically injected AAV into the cortex to induce the expression of EGFP. Because the 2.5 pseudotype exhibits preferential tropism to astrocytes (J. Dong, unpublished observations), we were able to readily observe individual astrocytes in brain sections. A volumetric analysis revealed similar results to those obtained with the dnSNARE animal: the volume of a single astrocyte was $\left(24,465 \mu \mathrm{m}^{3}\right)$ (supplemental Fig. 2, available at www.jneurosci.org as supplemental material). Together, this volumetric analysis shows that cortical astrocytes are organized in a nonoverlapping manner in agreement with the tiling organization demonstrated previously in the hippocampus (Bushong et al., 2002).

\section{Single astrocytes enwrap multiple neuronal cell bodies}

We observed that cortical astrocytes in dnSNARE sections harbored several holes (Figs. $2 A, B$, left). We suspected that these holes were filled with neuronal somata, which we confirmed by NeuN staining (Figs. $2 A, B$, right). We then determined the number of neuronal somata enwrapped by a single astrocyte (see Ma- 
terial and Methods). Using the volume of a single astrocyte obtained in our previous analysis to identify single astrocytes, we observed that on average four neuronal somata were enwrapped by a single astrocyte (Fig. 2D) with an upper limit of eight. To address potential concerns about the expression of dnSNARE within astrocytes affecting this structural relationship, we asked whether EGFP-positive astrocytes transduced with AAV exhibited this phenomenon. We found that AAVtransduced astrocytes also enwrap neuronal somata similar to the dnSNARE astrocytes (Fig. 2E-G).

To ask whether an astrocyte clusters neurons within its territory, we performed a nearest neuronal neighbor analysis on neuronal somata within the domain of single astrocytes. We found a slightly higher probability of a neuron having as its nearest neuronal neighbor located within the same astrocytic territory $(62.77 \pm 5.01 \%)$ (supplemental Fig. 3, available at www. jneurosci.org as supplemental material). The average distance between a neuronal cell body and its nearest neuronal neighbor was $11.16 \pm 0.39 \mu \mathrm{m}$ when the neighbor was within the territory of the astrocyte compared with $12.9 \pm 0.49 \mu \mathrm{m}$ when it was outside.

\section{Single astrocytes are associated with up to 600 dendrites}

To study the three-dimensional relationship between single astrocytes and single neurons, we filled cortical neurons of the transgenic animals with biocytin through a patch pipette. Subsequent staining of biocytin with an Alexa 546-conjugated streptavidin allowed us to visualize the fine neuronal morphology and study its structural relationship with the EGFPexpressing astrocytes. Single astrocytes were identified based on the volume analysis performed in Figure 1. Figure $3 A$ shows that the dendritic tree of a neuron requires numerous astrocytes for complete coverage. Because the entire dendritic tree of a neuron is not contained within one brain slice we did not attempt

to estimate this number. Instead, we estimated the number of dendritic spines influenced by one astrocyte. Of the nine layer $2 / 3$ neurons that were filled, only four were located in a region where single EGFP+ astrocytes could be identified based on the volume analysis described in Figure 1. From these four neurons, the average length of basal dendrites (within $200 \mu \mathrm{m}$ from the cell somata) that spanned through a single astrocyte was $50.8 \pm 3.3$ $\mu \mathrm{m}(n=24)$ (Fig. $3 B)$. The average linear spine density in the basal dendrites of the nine neurons was $0.7 \pm 0.1$ spine $/ \mu \mathrm{m}$ (Fig. $3 C, D)$. Thus, on average, a single astrocyte has the potential to contact 36 spines per dendrite.

Because the density of synapses in cortex has been estimated to
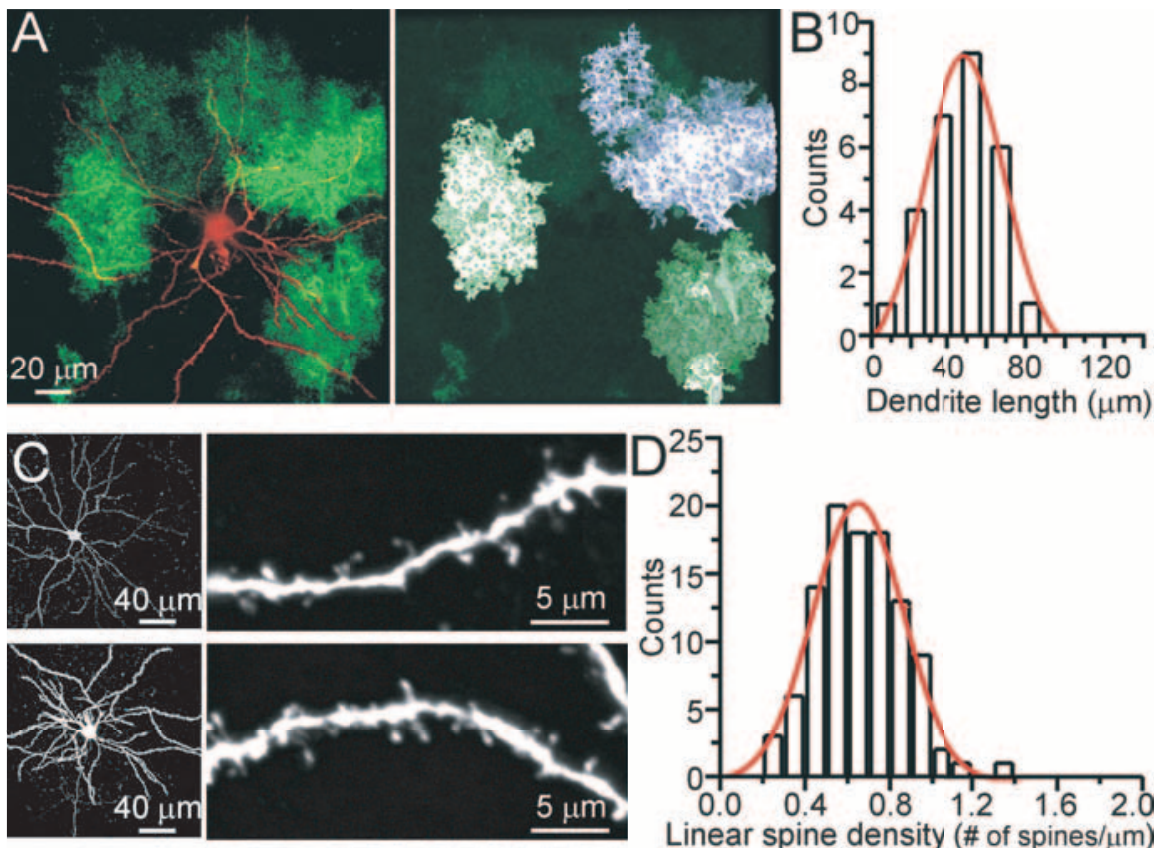

Dendrite length $(\mu \mathrm{m})$

Figure 3. Single astrocytes enwrap different dendrites of the same neuron. $\boldsymbol{A}$, Left, Top-view reconstruction showing a 作 Higher-magnification images allow the identification of single spines. $\boldsymbol{D}$, Distribution of the linear density of spines measured in 105 dendrite segments from nine biocytin-filled cortical neurons.
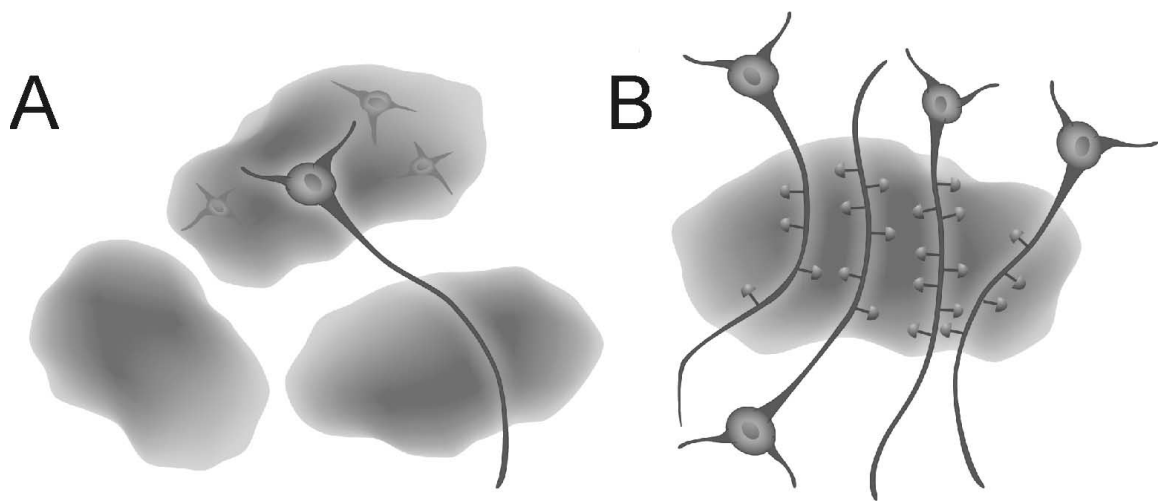

Figure 4. Schematic representation of functional synaptic islands. $\boldsymbol{A}$, Diagram showing three astrocytes. The holes are filled with neurons, one of which is shown to have its processes extending out to other astrocytes pointing to the potential of different neuronal compartments being modulated by different astrocytes. $\boldsymbol{B}$, Diagram illustrating the concept of functional synaptic islands: a group of dendrites from several neurons are enwrapped by a single astrocyte. Synapses localized within the territory of this astrocyte have the potential to be modulated in a coordinated manner by gliotransmitter(s) released from this glial cell.

be one per cubed micrometer (Genoud et al., 2004) and the volume of a single astrocyte is $23,000 \mu \mathrm{m}^{3}$ (Fig. 1), it is possible to estimate the number of dendrites contacted by one astrocyte. Because the average dendritic length within an astrocytic domain is $50 \mu \mathrm{m}$ with a linear spine density of 0.7 spines/ $\mu \mathrm{m}$, we can estimate that up to 600 dendrites are located within an astrocyte working on the assumption that the majority of synapses are formed on spines and that each spine represents a single synapse. Other studies of linear spine density have estimated a density of 1-1.5 spines/ $\mu \mathrm{m}$ (Ballesteros-Yanez et al., 2006). Because they used a light microscopy approach, it is likely that they were able to count spines within the $z$ plane that we may have missed in our 
analysis. Thus, between 300 and 600 dendrites are associated within the domain of a single astrocyte.

\section{Discussion}

We propose that the microvolumes of the nervous system occupied by individual astrocytes have the potential to act as nodes of synaptic modulation and coordination (Fig. 4). Because we are beginning to appreciate that $\mathrm{Ca}^{2+}$ signals are normally restricted to individual astrocytes in vivo (Wang et al., 2006) rather than as intercellular propagating $\mathrm{Ca}^{2+}$ waves previously identified in vitro (Guthrie et al., 1999), it becomes possible to speculate about functional interactions between individual astrocytes and neurons. Assuming that second-messenger signaling within astrocytes is normally a compartmentalized process that is restricted within glia, the release of gliotransmitters from individual astrocytes has the potential to influence the synapses associated with hundreds of dendrites. Previously, we have shown that the stimulation of presynaptic afferents activates synaptically associated astrocytes, which in turn release ATP leading to the accumulation of adenosine (Pascual et al., 2005). This activity-dependent accumulation of adenosine, in turn, causes a presynaptic inhibition and heterosynaptic depression of neighboring synapses. Thus, one could readily envision that the synapses associated with one astrocyte could be modulated in this manner, whereas synapses of neighboring astrocytic domains are not. Cortical astrocytes are coupled by gap junctions (Nimmerjahn et al., 2004), raising the potential for communication between synaptic nodes supported by diffusion of molecules between neighboring astrocytes through these structures. The significance of such a relatively slow process in influencing synaptic transmission and plasticity remains to be determined.

In conclusion, we have determined that, in agreement with a previous study performed in the hippocampus, that there is little overlap in the spatial territories occupied by individual astrocytes. A given astrocyte directly interacts with an average of four cortical neuron cell bodies and this same astrocyte has associated with its processes between 300 and 600 dendrites, the majority of which are likely to be derived from distinct parent neuronal cell bodies. Because $\mathrm{Ca}^{2+}$ signals are generally restricted within individual astrocytes and induce the release of gliotransmitters, we propose the concept of functional islands of synapses in which neighboring synapses can be coordinated by signals derived from individual glia (Fig. 4) (Pascual et al., 2005).

\section{References}

Angulo MC, Kozlov AS, Charpak S, Audinat E (2004) Glutamate released from glial cells synchronizes neuronal activity in the hippocampus. J Neurosci 24:6920-6927.

Arcuino G, Lin JH, Takano T, Liu C, Jiang L, Gao Q, Kang J, Nedergaard M
(2002) Intercellular calcium signaling mediated by point-source burst release of ATP. Proc Natl Acad Sci USA

Ballesteros-Yanez I, Benavides-Piccione R, Elston GN, Yuste R, DeFelipe J (2006) Density and morphology of dendritic spines in mouse neocortex. Neuroscience 138:403-409.

Bezzi P, Carmignoto G, Pasti L, Vesce S, Rossi D, Rizzini BL, Pozzan T, Volterra A (1998) Prostaglandins stimulate calcium-dependent glutamate release in astrocytes. Nature 391:281-285.

Bushong EA, Martone ME, Jones YZ, Ellisman MH (2002) Protoplasmic astrocytes in CA1 stratum radiatum occupy separate anatomical domains. J Neurosci 22:183-192.

Fellin T, Pascual O, Gobbo S, Pozzan T, Haydon PG, Carmignoto G (2004) Neuronal synchrony mediated by astrocytic glutamate through activation of extrasynaptic NMDA receptors. Neuron 43:729-743.

Fiacco TA, McCarthy KD (2004) Intracellular astrocyte calcium waves in situ increase the frequency of spontaneous AMPA receptor currents in CA1 pyramidal neurons. J Neurosci 24:722-732.

Genoud C, Knott GW, Sakata K, Lu B, Welker E (2004) Altered synapse formation in the adult somatosensory cortex of brain-derived neurotrophic factor heterozygote mice. J Neurosci 24:2394-2400.

Guthrie PB, Knappenberger J, Segal M, Bennett MV, Charles AC, Kater SB (1999) ATP released from astrocytes mediates glial calcium waves. J Neurosci 19:520-528.

Kang J, Jiang L, Goldman SA, Nedergaard M (1998) Astrocyte-mediated potentiation of inhibitory synaptic transmission. Nat Neurosci 1:683-692.

Kozlov AS, Angulo MC, Audinat E, Charpak S (2006) Target cell-specific modulation of neuronal activity by astrocytes. Proc Natl Acad Sci USA 0603741103.

Liu QS, Xu Q, Arcuino G, Kang J, Nedergaard M (2004) Astrocyte-mediated activation of neuronal kainate receptors. Proc Natl Acad Sci USA 101:3172-3177.

Nimmerjahn A, Kirchhoff F, Kerr JN, Helmchen F (2004) Sulforhodamine 101 as a specific marker of astroglia in the neocortex in vivo. Nat Methods 1:31-37.

Panatier A, Theodosis DT, Mothet JP, Touquet B, Pollegioni L, Poulain DA, Oliet SH (2006) Glia-derived D-serine controls NMDA receptor activity and synaptic memory. Cell 125:775-784.

Parpura V, Basarsky TA, Liu F, Jeftinija K, Jeftinija S, Haydon PG (1994) Glutamate-mediated astrocyte-neuron signalling. Nature 369:744-747.

Pascual O, Casper KB, Kubera C, Zhang J, Revilla-Sanchez R, Sul JY, Takano H, Moss SJ, McCarthy K, Haydon PG (2005) Astrocytic purinergic signaling coordinates synaptic networks. Science 310:113-116.

Schell MJ, Molliver ME, Snyder SH (1995) D-serine, an endogenous synaptic modulator: localization to astrocytes and glutamate-stimulated release. Proc Natl Acad Sci USA 92:3948-3952.

Serrano A, Haddjeri N, Lacaille JC, Robitaille R (2006) GABAergic network activation of glial cells underlies hippocampal heterosynaptic depression. J Neurosci 26:5370-5382.

Ventura R, Harris KM (1999) Three-dimensional relationships between hippocampal synapses and astrocytes. J Neurosci 19:6897-6906.

Wang X, Lou N, Xu Q, Tian GF, Peng WG, Han X, Kang J, Takano T, Nedergaard M (2006) Astrocytic $\mathrm{Ca}^{2+}$ signaling evoked by sensory stimulation in vivo. Nat Neurosci 9:816-823.

Zhang JM, Wang HK, Ye CQ, Ge W, Chen Y, Jiang ZL, Wu CP, Poo MM, Duan S (2003) ATP released by astrocytes mediates glutamatergic activity-dependent heterosynaptic suppression. Neuron 40:971-982. 